\title{
Characteristic Timescales of the Local Moment Dynamics in Hund's-metals
}

\author{
C. Watzenböck ${ }^{a}$, M. Edelmann ${ }^{b}$, D. Springer ${ }^{a}$, G. Sangiovanni ${ }^{b}$, and A. Toschi ${ }^{a}$ \\ ${ }^{a}$ Institute of Solid State Physics, TU Wien, 1040 Vienna, Austria and \\ ${ }^{b}$ Institut für Theoretische Physik und Astrophysik and Würzburg-Dresden Cluster of Excellence ct.qmat, \\ Universität Würzburg, 97074 Würzburg, Germany
}

(Dated: August 10, 2020)

\begin{abstract}
We study the characteristic timescales of the fluctuating local moments in Hund's metal systems for different degrees of correlation. By analyzing the dynamical spin susceptibility in the real-time domain we determine the timescales controlling oscillation and damping of on-site fluctuations - a crucial factor for the detection of local moments with different experimental probes. We apply this procedure to different families of iron pnictides/chalcogenides, explaining the material trend in the discrepancies reported between experimental and theoretical estimates of their magnetic moments.
\end{abstract}

PACS numbers: 71.27.+a, 75.20.Hr, 71.10.Fd

Introduction. - Our perception of the natural world is significantly shaped by the properties of the detection process considered. One crucial aspect is the timescale of the probing mechanism: If this is larger than the typical timescale of the phenomenon under investigation, only averaged information will be gained. This general statement applies to a very broad class of detectors, ranging, e.g. from the vision process in our eyes to the case of interest for this work: the measurement of magnetic properties in correlated materials.

Here, we focus on the detection of the local magnetic moments in correlated metallic systems. Their proper description is, indeed, a key to understanding manyelectron systems beyond the conventional band-theory framework, being central to: Kondo physics[1, 2], MottHubbard[3-5] or Hund-Mott[6-10] metal-insulator transitions, quantum criticality of heavy fermion systems[11, 12], magnetic and spectroscopic properties of $\mathrm{Ni}$ and $\mathrm{Fe}[13-15]$ and of unconventional superconductors[16, 17].

Reflecting the high physical interest, several experimental procedures are used to detect the local magnetic moments and their manifestations[18]: measurements of static susceptibilities [13, 18], inelastic neutron spectroscopy (INS) [19], by integrating over the Brillouin zone(BZ) [20], x-ray absorption or emission spectroscopy (XAS or XES), etc.

Whether it is possible to obtain an accurate description of the local moments largely depends on the relation between the intrinsic timescales of the experimental probes and those characterizing the dynamical screening mechanisms at work. The emerging picture is typically clear-cut if the screening processes are strongly suppressed: In Mott or Hund's-Mott insulating phases coherent description of the magnetic moment properties can be easily obtained in all experimental setups. A more complex, multifaceted situation characterizes systems where well preformed magnetic moments present a rich dynamics. Good examples are the strongly correlated metallic regimes adjacent to a Mott metalinsulator transition, or even better, compounds display- ing a Hund's metal behavior[6, 21], such as iron pnictides and chalcogenides[17].

In this work, we illustrate how to quantitatively estimate the characteristic timescales of fluctuating moments in many-electron systems within the regime of linear response. As a pertinent example, we apply this procedure to investigate the puzzling discrepancies between experimental and theoretical estimates of the magnetic moment size in the different families of iron pnictides/chalcogenides, clarifying the peculiar material dependence of this long-standing issue.

An intuitive picture. - For a transparent interpretation of our realistic calculations, we start from some heuristic considerations on the dynamics of the local magnetic moment $\vec{\mu}=g \frac{\mu_{B}}{\hbar} \vec{S}$ in a correlated metal. The relevant information is encoded in the time dependence of its correlation function

$$
\mathcal{F}(t) \equiv \frac{1}{2} g^{2} \frac{\mu_{B}^{2}}{\hbar^{2}}\left\langle\left\{\hat{S}_{z}(t), \hat{S}_{z}(0)\right\}\right\rangle,
$$

where $g \cong 2$ is the Landé factor, $\mu_{B}$ the Bohr magneton and $\hat{S}_{z}=\sum_{\ell} \hat{s}_{z}^{\ell}$ the $z$-component of the total spin moment hosted by the correlated atom (e.g., a transition metal element), built up by the unpaired electronic spins $s_{z}$ of its partially filled $d$ or $f$ shells [18]. We stress that Eq. (1) describes both the static (thermal) and dynamic (Kubo) part of the response [22], which is needed for our study. In general, one expects the maximum values of $\mathcal{F}(t)$ at $t=0$ : This describes the instantaneous spin configuration of the system, often quite large in a multiorbital open shell due to the Hund's rule. Because of electronic fluctuations, the probability of finding a magnetic moment of the same size and the same orientation will be decreasing with time. At a first approximation, one can identify two distinct patterns for this process: (i) a gradual rotation (with constant amplitude) and (ii) a progressive reduction of the size of the local moment. Within this simple picture, two characteristic time (and energy) scales for the local moment dynamics are naturally defined: (i) the period of the rotation $\left(t_{\bar{\omega}} \propto \frac{1}{\bar{\omega}}\right)$ and (ii) the characteristic time $\left(t_{\gamma} \propto \frac{\hbar}{\gamma}\right)$ for the amplitude 


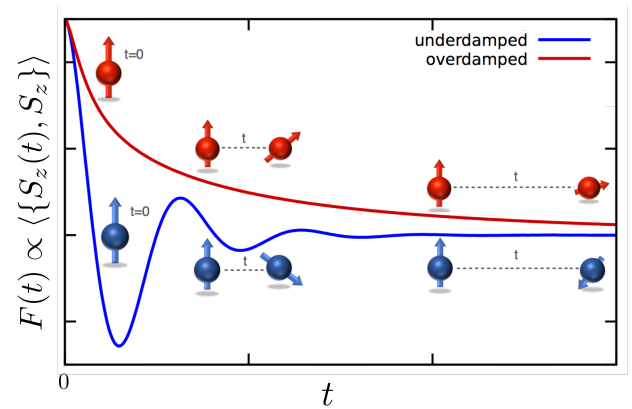

FIG. 1. Schematic representation of the time decay of local spin correlations in the underdamped/overdamped regimes.

damping.

The values of the characteristic timescales may vary considerably from one material to another, with overall larger values associated to a suppressed electronic mobility. In the extreme case of a Mott insulator, one expects to observe long-living magnetic moments, consistent with the analytic divergence of the timescales found in the fully localized (atomic) limit $\left(t_{\bar{\omega}}, t_{\gamma} \rightarrow \infty\right)$. On the opposite side, in a conventional (weakly correlated) metal both scales will be extremely short, roughly of the order of the inverse of the bandwidth $W$ of the conducting electrons $\left(t_{\bar{\omega}} \sim t_{\gamma} \propto \frac{\hbar}{W}\right)$. The most interesting situation is realized in a correlated metallic context. Here, the slowing down of the electronic motion, induced by the electronic scattering, increases the values of both timescales that remain finite, nonetheless. The enhancement will depend on specific aspects of the many-electron problem considered, possibly affecting the two timescales in a different fashion: This leads to the distinct regimes of underdamped $\left(t_{\gamma} \gg t_{\bar{\omega}}\right)$ and overdamped $\left(t_{\gamma} \ll t_{\bar{\omega}}\right)$ local moment fluctuations, schematically depicted in Fig. 1. The actual hierarchy of the timescales will strongly impact the outcome of spectroscopic experiments. Further, quantitative information about the dynamics of the magnetic fluctuations at equilibrium may also provide important information for the applicability of the adiabatic spin dynamics[23-25] and, on a broader perspective, crucial insights for the highly nontrivial interpretation of the out-of-equilibrium spectroscopies.

Quantification of timescales. - The procedure to quantitatively estimate the characteristic timescales from many-electron calculations and/or experimental measurements relies on the Kubo-Nakano formalism for linear response. Here, we recall that the dynamical susceptibility is defined as

$$
\chi(\tau) \equiv\left\langle T_{\tau} \hat{S}_{z}(\tau) \hat{S}_{z}(0)\right\rangle
$$

in imaginary time $\left(T_{\tau}\right.$ is the imaginary time-ordering operator). The corresponding (retarded) spectral functions $\chi^{R}(\omega)$ are obtained via analytic continuation of Eq. (2). The absorption component of the spectra, $\operatorname{Im} \chi^{R}(\omega)$, directly measurable (e.g. in INS), provides a direct route for quantifying the timescales. In particular, simple analytic expressions, directly derived for damped harmonic oscillators, can be exploited for fitting the (one or more) predominant absorption peak(s) of $\operatorname{Im} \chi^{R}(\omega)$. In the illustrative case discussed above, one has

$$
\operatorname{Im} \chi^{R}(\omega)=A \frac{2 \gamma \omega}{\left(\omega^{2}-\omega_{0}^{2}\right)^{2}+4 \omega^{2} \gamma^{2}},
$$

where $\gamma$ and $\omega_{0}$ are the scales associated to the major absorption processes active in the system under consideration (with $\hbar=1$ ), and the constant $A$ reflects the size of the instantaneous magnetic moment. The expression is clearly generalizable to other cases, where more absorption peaks are visible in the spectra, as a sum of the corresponding contributions [26].

The full time-dependence of the fluctuating local moment, which will reflect the interplay of the timescales defined above, is eventually obtained via the fluctuationdissipation theorem

$$
\mathcal{F}(t)=\frac{1}{\pi} \int_{0}^{\infty} \mathrm{d} \omega \cos (\omega t) \operatorname{coth}(\beta / 2 \omega) \operatorname{Im} \chi^{R}(\omega),
$$

where $\beta=\left(k_{B} T\right)^{-1}$ is the inverse temperature.

The case of the Hund's-metals. - While the procedure illustrated above is applicable to all spectroscopic experiments of condensed matter systems, we will demonstrate its advantages for studying Hund's metals[6, 21], where the dynamics of fluctuating moments is of particular interest [27]. These systems can be viewed as a new "crossover"-state of matter, triggered by sizable values of the local Hubbard repulsion $(U)$ and Hund's rule coupling $(J)$, when the corresponding atomic shell is (about) one electron away from a half-filled multiorbital configuration. At strong coupling, the interplay between $U$ and $J$ can induce either a Mott or a charge-disproportionate Hund's insulator $[9,28]$. Out of half-filling, the competition between these two tendencies can also stabilize a metallic ground state in the presence of high values of the electronic interaction $[6,9,28,29]$. The emerging physics of a large local magnetic moment fluctuating in a strongly correlated metallic surrounding evidently represents one of the best playgrounds to applying our time-resolved procedure.

The prototypical class of materials displaying Hund's metal physics is represented by the iron pnictides or chalcogenides. These compounds, which often display unconventional superconducting phases upon doping, are also characterized by interesting magnetic properties $[17,20,30]$. Both the ordered magnetic moments (measured by neutron diffraction in the magnetically ordered phase) and the fluctuating moments (measured by INS in the paramagnetic high- $T$ phase) are reported to be systematically lower[31] in experiment than in (static) local spin density approximation (LSDA) calculations (predicting a large ordered moment of about $2 \mu_{B}$ for almost all compounds of this class). It was also noted that, 
Bare Bubble
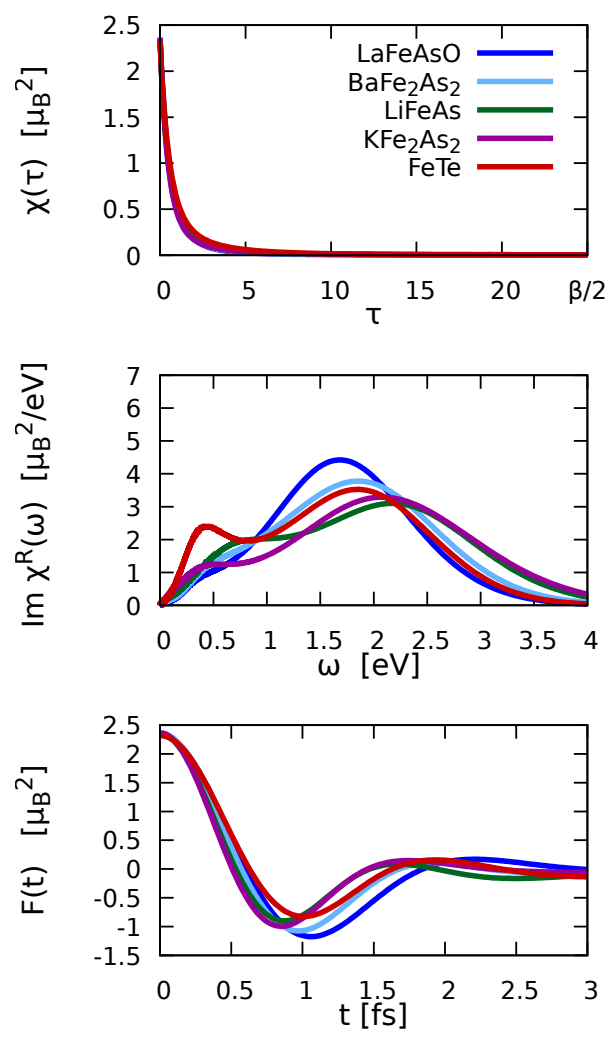

DMFT Bubble
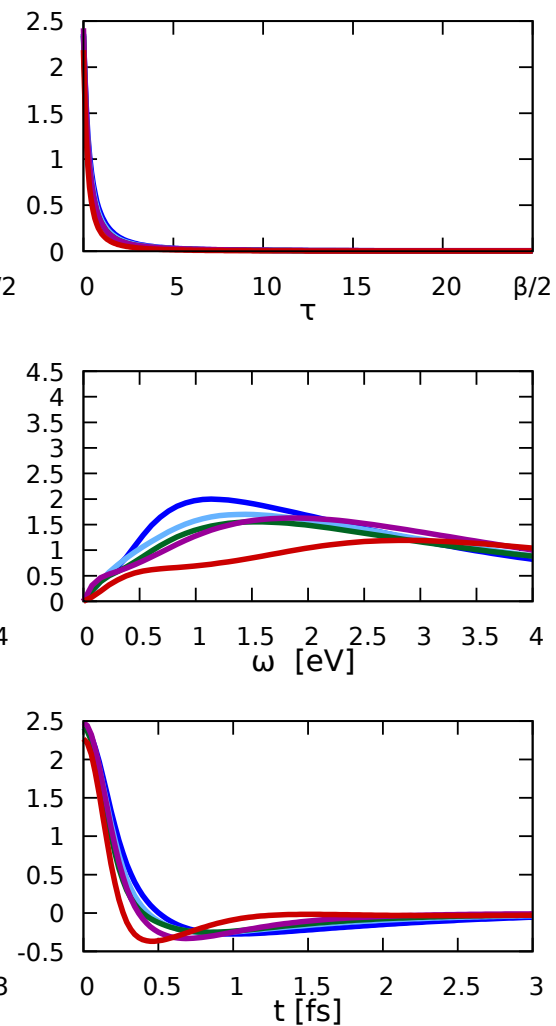

Full Calculation
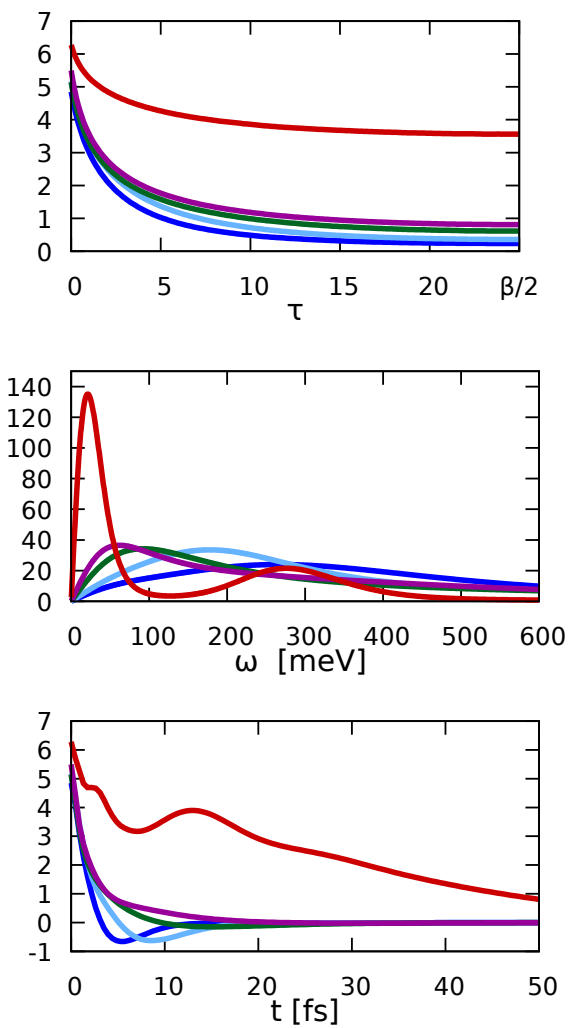

FIG. 2. Spin susceptibility of the $3 d$-Fe atoms as a function of imaginary time (first row), corresponding absorption spectra in real frequency (second row) and correlation function in real time (third row), computed for different families of iron pnictides or chalcogenides at $\beta=50 \mathrm{eV}^{-1}(T \approx 232 \mathrm{~K})$ in the DFT+DMFT (third column), compared with the corresponding results of the bare (first column) and the DMFT (second column) bubble calculations.

surprisingly, the larger discrepancies are found for the "less correlated" families 1111 (e.g. LaFeAsO) and 122 (e.g. $\mathrm{BaAs}_{2} \mathrm{O}_{2}$ ), which display milder quasiparticle renormalization effects and are characterized by lower values of the screened Coulomb interaction estimated in constrained random phase approximation (cRPA)[32]. Significantly smaller (or almost no) deviations are reported, instead, for the most correlated families such as the 11 subclass (e.g. FeTe), where relatively large local moments are found both in neutron experiments and theory. Previous dynamical mean-field theory (DMFT) studies of the INS results suggested[33-36] that the local spin fluctuations on the Fe atom-whose time-resolved description is the central topic here-may be responsible for the observed discrepancies. These works were restricted to one compound or (at most) one family only, and did not analyze the real-time domain. Hence, no definitive conclusion could be drawn about this issue, motivating the present computational material study.

Ab-initio + DMFT calculations. - We report here on our density functional theory (DFT) + DMFT calculations $[37,38]$ of the local spin susceptibilities in the iron pnictides/chalcogenides. Different from preceding works, we computed the spin-spin response functions on equal footing for several different compounds, chosen as representative of the most relevant families $(1111,122,111$, 11). As a step forward in the theoretical description, we put emphasis on a quantitative time-resolved analysis of the results, eventually allowing for a precise interpretation of the physics at play and of the spectroscopic results.

For our DMFT calculations[26, 39], we considered a projection on the Fe-3d (maximally localized) Wannierorbital manifold. We assume an on-site electrostatic interaction with a generalized (orbital-dependent) Kanamori form. The corresponding Hamiltonian reads:

$$
H=\sum_{\mathbf{k} \sigma l m} H_{l m}(\mathbf{k}) c_{\mathbf{k} l \sigma}^{\dagger} c_{\mathbf{k} m \sigma}+H_{\mathrm{int}}
$$

where $l, m$ are orbital indices, $\mathbf{k}$ denotes the fermionic 


\begin{tabular}{lllllll}
\hline & $\omega_{0}[\mathrm{eV}]$ & $\gamma[\mathrm{eV}]$ & $t_{\gamma}[\mathrm{fs}]$ & $t_{\bar{\omega}}[\mathrm{fs}]$ & & $\frac{t_{1 \mathrm{P}}[\mathrm{fs}]}{2}$ \\
\cline { 1 - 5 } $\mathrm{LaFeAsO}$ & $\mathbf{0 . 3 9}$ & 0.35 & 1.9 & 3.8 & & 30.80 \\
$\mathrm{BaFe}_{2} \mathrm{As}_{2}$ & $\mathbf{0 . 2 8}$ & $\mathbf{0 . 2 8}$ & 2.4 & 15.2 & & 19.96 \\
$\mathrm{LiFeAs}$ & 0.30 & $\mathbf{0 . 5 8}$ & 7.9 & - & 12.23 \\
$\mathrm{KFe}_{2} \mathrm{As}_{2}$ & 0.51 & $\mathbf{2 . 0 8}$ & 10.3 & - & 9.08 \\
$\mathrm{FeTe}$ & $\mathbf{0 . 0 2 9}$ & 0.022 & 29.3 & 34.8 & 2.14 \\
\hline
\end{tabular}

TABLE I. Fitting parameters $\omega_{0}$ and $\gamma$ of the absorption peak(s) computed in DMFT with Eq. (3) (first and second column, where the largest energy scale is marked in bold); effective lifetime $\chi(t \rightarrow \infty) \propto \mathrm{e}^{-t / t_{\gamma}}$ (third column); effective oscillation period $t_{\bar{\omega}}=\hbar / \sqrt{\omega_{0}^{2}-\gamma^{2}}$ (fourth column) and $t_{1 \mathrm{P}}=\left\langle\hbar / 2 Z_{i} \operatorname{Im} \Sigma_{\mathrm{i}}(\omega \rightarrow 0)\right\rangle_{\text {all orb. (fifth column) is the effec- }}$ tive orbital averaged one-particle lifetime for the different material considered. See [26] for further details.

momentum, and $\sigma, \sigma^{\prime}$ the spin, and

$$
\begin{aligned}
H_{\mathrm{int}} & =\sum_{\mathbf{r} l} U_{l l} n_{\mathbf{r} l \uparrow} n_{\mathbf{r} l \downarrow} \\
& +\sum_{\mathbf{r} \sigma \sigma^{\prime}, l<m}\left(U_{l m}-J_{l m} \delta_{\sigma \sigma^{\prime}}\right) n_{\mathbf{r} l \sigma} n_{\mathbf{r} m \sigma^{\prime}} \\
& -\sum_{\mathbf{r}, l \neq m} J_{l m}\left[c_{\mathbf{r} l \uparrow}^{\dagger} c_{\mathbf{r} l \downarrow}^{\dagger} c_{\mathbf{r} m \uparrow} c_{\mathbf{r} m \downarrow}+c_{\mathbf{r} l \uparrow}^{\dagger} c_{\mathbf{r} m \downarrow}^{\dagger} c_{\mathbf{r} m \uparrow} c_{\mathbf{r} l \downarrow}\right]
\end{aligned}
$$

where $\mathbf{r}$ indicates the lattice site, and the realistic values of the screened electrostatic interactions $U_{l m}$ and $J_{l m}$ for the different materials have been taken from Ref.[32], as detailed in [26]. The orbitally averaged values of $\bar{U}, \bar{J}$ range from $(2.53,0.38) \mathrm{eV}$ for $\mathrm{LaFeAsO}$ to $(3.41,0.48) \mathrm{eV}$ for FeTe [26].

Our DMFT results are summarized in Fig. 2, where we show the dynamical spin susceptibility on the Fe atoms of all compounds considered in its different representations: imaginary time in the first-row panels [cf. Eq. (2)] which is the direct output[40] of the quantum Monte Carlo (QMC) solver, real-frequency in the second row [from analytic continuations], real-time in the third row [Eq. (1), via Eq. (4)]. In all cases, we performed our analysis not only for the full DMFT calculation (third column panels), which comprises - per construction- all purely local effects[41, 42] of the DMFT self-energy and vertex corrections, but we also evaluate, separately, the corresponding "bubble" terms (i.e., $\chi_{0}=-\beta G G$ ) either computed with the noninteracting Green's function $\left(G=G_{0}\right.$, first column) or with the DMFT one $\left(G=G_{\mathrm{DMFT}}\right.$, i.e. by including the DMFT self-energy, second column).

A quick glance at $\chi(\tau)$ already illustrates an important finding of our work: The different band structure of the materials as well as their self-energies does not generate by itself any distinguishable effects in the local moment dynamics (first two columns in Fig. 2). Instead, the definite material dependence observed is almost totally originated by vertex corrections (third column).

One can understand the overall material trend as fol-

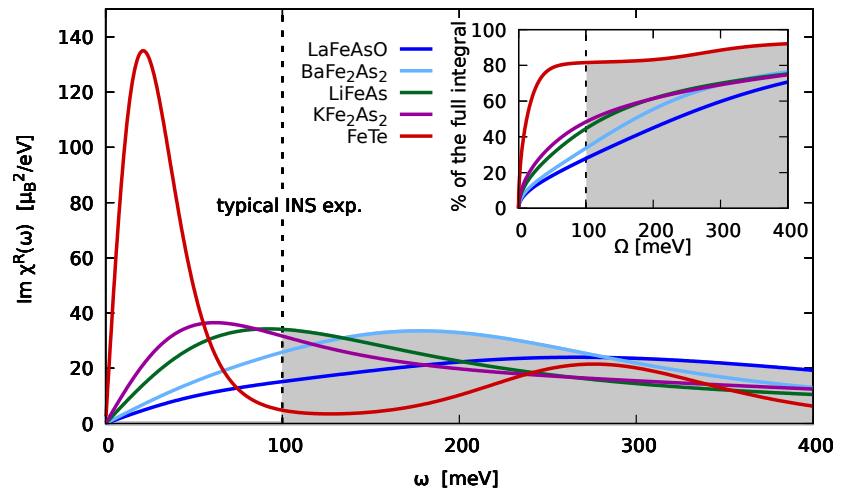

FIG. 3. Material dependence of the spin-absorption spectra in the different families of the iron pnictides/chalchogenides computed in DFT+DMFT, compared with the typical energy threshold $(\sim 100 \mathrm{meV})$ of INS experiments. Inset: Corresponding fraction of $m_{\text {loc }}^{2}$ obtained integrating Eq. (7) up to $\Omega$.

lows: Instantaneous $(\tau=0)$ magnetic moments of similar (and large) sizes but subjected to quite different screening effects $\left(\tau \rightarrow \frac{\beta}{2}\right)$. However, only the corresponding analysis of $\operatorname{Im} \chi^{R}(\omega)$ and $\mathcal{F}(t)$ allows to extract clearcut physical information. By looking at the data for $\mathcal{F}(t)$, we easily note that the moment dynamics described by the "bubble terms" (with or without $\Sigma_{\mathrm{DMFT}}$ ) is controlled by very short timescales for oscillation and damping $(\sim 0.5 \mathrm{fs})$, roughly corresponding to $\sim \hbar / W$. The inclusion of vertex corrections causes, instead, a significant and strongly material-dependent slowing down of the dynamics: In the "least-correlated" LaFeAsO, we already observe oscillation and damping over few fs (one order of magnitude larger than in the noninteracting case). These timescales visibly increase considering more correlated families, up to the extreme case of FeTe, dominated by an extremely long decay over more than $25 \mathrm{fs}$.

The scenario emerging from the visual inspection of $\mathcal{F}(t)$ is supported, at a quantitative level, by the fit of the main absorption peaks of $\operatorname{Im} \chi^{R}(\omega)$, see Tab. I for details. The values $t_{\gamma}$ and $t_{\bar{\omega}}$ range from 3 to $30 \mathrm{fs}$, with an overall trend which trails the progressive reduction of the quasiparticle life time $\left(t_{1 P}\right)$ across the different families.

Spectroscopic measurements. - The significant spread of the estimated timescale values directly affect the detectability of the local magnetic moments $\left(m_{\mathrm{loc}}\right)$ in the iron pnictides or chalcogenides. While fast probes (e.g. XAS, XES) are able to detect the high-spin instantaneous configuration of these Hund's metals, the characteristic timescale of the INS $\left(t_{\mathrm{INS}} \simeq 5-10 \mathrm{fS} \simeq \hbar / E_{\mathrm{INS}}\right.$, with $E_{\mathrm{INS}}=\hbar \Omega_{\mathrm{INS}} \simeq 100 \mathrm{meV}$ [43]) are of the same order as those in Tab. I: time-averaging effects will, thus, lead to 
underestimate the local magnetic moment:

$$
\begin{aligned}
m_{\mathrm{loc}}^{2} & =\frac{3}{\pi} \lim _{\Omega \rightarrow \infty} \frac{\int_{-\Omega}^{\Omega} \int_{\mathrm{BZ}} \operatorname{Im} \chi^{R}(\vec{q}, \omega) b(\omega) \mathrm{d} \vec{q} \mathrm{~d} \omega}{\int_{\mathrm{BZ}} \mathrm{d} \vec{q}} \\
& =\frac{3}{\pi} \lim _{\Omega \rightarrow \infty} \int_{-\Omega}^{\Omega} \operatorname{Im} \chi_{\mathrm{loc}}^{R}(\omega) b(\omega) \mathrm{d} \omega,
\end{aligned}
$$

where $b(\omega)=1 /\left(\mathrm{e}^{\beta \omega}-1\right)$ is the Bose-Einstein distribution function (with $\hbar=1$ ). This is especially relevant for the "less correlated" compounds (LaFeAsO and $\mathrm{BaFe}_{2} \mathrm{As}_{2}$ ), where $t_{\gamma}, t_{\bar{\omega}}<t_{\mathrm{INS}}$. In families with higher degrees of (e.g. for FeTe, where $t_{\gamma}, t_{\bar{\omega}}>t_{\mathrm{INS}}$ ) the averaging effect gets "mitigated", allowing the detection of larger magnetic moment sizes, consistent with fast probe XAS and XES experiments[44, 45]. The material dependence of local moment dynamics is directly mirrored in the progressive red shift of the first-absorption peak in $\operatorname{Im} \chi^{R}(\omega)$, as shown in Fig. 3. Here, one can appreciate how an increasing part of the spin absorption spectra gradually enters the accessible energy window of the INS (main panel). This explains the progressively reduced discrepancies in the size of the magnetic moment (see inset) observed in the more correlated families of the iron pnictides or chalcogenides.

Conclusions. - We illustrated how to quantitatively investigate, on the real-time domain, the dynamics of magnetic moments in correlated systems and how to physically interpret the obtained results in terms of their characteristic timescales. Our procedure, exploiting the fluctuation-dissipation theorem, is then applied to clarify the results of INS experiments in several families of iron pnictides and chalcogenides. In particular, the different degrees of discrepancies with respect to the standard $a b$ initio calculations is rigorously explained by comparing the timescales of the fluctuating moments to the characteristic timescale of the INS probe. Remarkably, the strong differentiation among the timescales of the materials considered, crucial for a correct understanding of the underlying physics, is almost entirely due to vertex corrections.

While the dynamics of the magnetic moments is particularly intriguing in the Hund's metal materials considered here, the same procedure is directly applicable to all many-electron systems and to fluctuations of different kinds[2]. A precise quantification of the characteristic timescales may provide new keys to connect the findings of equilibrium and out-of-equilibrium spectroscopies, as well as crucial information on the applicability of adiabatic spin dynamics approaches[25].

Acknowledgments. We thank B. Andersen, L. Boeri, M. Capone, L. de' Medici, P. Hansmann, K. Held, J. Tomczak and M. Zingl for insightful discussions. We acknowledge support by the Austrian Science Fund (FWF) through Projects SFB F41 (C.W., D.S.) and No. I 2794-N35 (A.T.) as well as by the Deutsche Forschungsgemeinschaft (DFG, German Research Foun- dation) through Grant No. SFB 1170, Projects No. 258499086 and No. EXC 2147, Project No. 390858490 "ct.qmat" (G.S.). Calculations were performed on the Vienna Scientific Cluster (VSC).

[1] A. Hewson, The Kondo Problem to Heavy Fermions (Cambridge University Press, 1993).

[2] J. M. Tomczak, (2019), arXiv:1904.01346 [cond-mat.strel].

[3] M. Imada, A. Fujimori, and Y. Tokura, Rev. Mod. Phys. 70, 1039 (1998).

[4] A. Georges, G. Kotliar, W. Krauth, and M. J. Rozenberg, Rev. Mod. Phys. 68, 13 (1996).

[5] P. Hansmann, A. Toschi, G. Sangiovanni, T. SahaDasgupta, S. Lupi, M. Maresi, and K. Held, Phys. Status Solidi B 250, 1251 (2013).

[6] L. de' Medici, J. Mravlje, and A. Georges, Phys. Rev. Lett. 107, 256401 (2011).

[7] J. Mravlje, M. Aichhorn, and A. Georges, Phys. Rev. Lett. 108, 197202 (2012).

[8] A. J. Kim, H. O. Jeschke, P. Werner, and R. Valentí, Phys. Rev. Lett. 118, 086401 (2017).

[9] A. Isidori, M. Berović, L. Fanfarillo, L. de' Medici, M. Fabrizio, and M. Capone, Phys. Rev. Lett. 122, 186401 (2019).

[10] D. Springer, B. Kim, P. Liu, S. Khmelevskyi, M. Capone, G. Sangiovanni, C. Franchini, and A. Toschi, (2019), arXiv:1910.05151 [cond-mat.str-el].

[11] H. von Löhneysen, A. Rosch, M. Vojta, and P. Wölfle, Rev. Mod. Phys. 79, 1015 (2007).

[12] M. Brando, D. Belitz, F. M. Grosche, and T. R. Kirkpatrick, Rev. Mod. Phys. 88, 025006 (2016).

[13] J. Kübler, Theory of Itinerant Electron Magnetism, International Series of Monogr (OUP Oxford, 2000).

[14] A. I. Lichtenstein, M. I. Katsnelson, and G. Kotliar, Phys. Rev. Lett. 87, 067205 (2001).

[15] A. Hausoel, M. Karolak, E. Şaşıŏlu, A. Lichtenstein, K. Held, A. Katanin, A. Toschi, and G. Sangiovanni, Nature Communications 8, 16062 (2017).

[16] P. A. Lee, N. Nagaosa, and X.-G. Wen, Rev. Mod. Phys. 78, 17 (2006).

[17] A. Chubukov and P. J. Hirschfeld, Physics Today 68, 46 (2015), https://doi.org/10.1063/PT.3.2818.

[18] S. Blundell, Magnetism in Condensed Matter, Oxford Master Series in Condensed Matter Physics (OUP Oxford, 2001).

[19] R. S. Fishman, J. A. Fernandez-Baca, and T. Ramm, Spin-Wave Theory and its Applications to Neutron Scattering and THz Spectroscopy, 2053-2571 (Morgan and Claypool Publishers, 2018) pp. 2-1 to 2-14.

[20] P. Dai, Rev. Mod. Phys. 87, 855 (2015).

[21] K. Haule and G. Kotliar, New Journal of Physics 11, 025021 (2009).

[22] R. M. Wilcox, Phys. Rev. 174, 624 (1968).

[23] M. Sayad and M. Potthoff, New Journal of Physics 17, 113058 (2015).

[24] M. Sayad, R. Rausch, and M. Potthoff, EPL (Europhysics Letters) 116 (2016), 10.1209/02955075/116/17001.

[25] C. Stahl and M. Potthoff, Phys. Rev. Lett. 119, 227203 
(2017)

[26] for further details, see Supplemental Material, which also includes Refs. [46-65].

[27] P. Werner, E. Gull, M. Troyer, and A. J. Millis, Phys. Rev. Lett. 101, 166405 (2008).

[28] L. Fanfarillo and E. Bascones, Phys. Rev. B 92, 075136 (2015).

[29] S. Backes, H. O. Jeschke, and R. Valentí, Phys. Rev. B 92, 195128 (2015).

[30] L. Boeri, "Understanding novel superconductors with ab initio calculations," in Handbook of Materials Modeling (Springer, Cham, 2018).

[31] Y.-T. Tam, D.-X. Yao, and W. Ku, Phys. Rev. Lett. 115, 117001 (2015).

[32] T. Miyake, K. Nakamura, R. Arita, and M. Imada, Journal of the Physical Society of Japan 79, 044705 (2010).

[33] P. Hansmann, R. Arita, A. Toschi, S. Sakai, G. Sangiovanni, and K. Held, Phys. Rev. Lett. 104, 197002 (2010).

[34] M. Liu, L. W. Harriger, H. Luo, M. Want, R. A. Ewings, T. Guidi, H. Park, K. Haule, G. Kotliar, S. M. Hayden, and P. Dai, Nature Physics 8, 376 (2012).

[35] A. Toschi, R. Arita, P. Hansmann, G. Sangiovanni, and K. Held, Phys. Rev. B 86, 064411 (2012).

[36] M. Wang, C. Zhang, X. Lu, G. Tan, H. Luo, Y. Song, M. Wang, X. Zhang, E. A. Goremychkin, T. G. Perring, T. A. Maier, Z. Yin, K. Haule, G. Kotliar, and P. Dai, Nature Communications 4, 2874 (2013).

[37] G. Kotliar, S. Y. Savrasov, K. Haule, V. S. Oudovenko, O. Parcollet, and C. A. Marianetti, Rev. Mod. Phys. 78, 865 (2006).

[38] K. Held, Advances in Physics 56, 829 (2007).

[39] M. Wallerberger, A. Hausoel, P. Gunacker, A. Kowalski, N. Parragh, F. Goth, K. Held, and G. Sangiovanni, Computer Physics Communications 235, 388 (2019).

[40] We compute the dynamical spin-response of the auxiliary impurity model associated to the self-consistent DMFTsolution, corresponding to the momentum-averaged one in the limit of high-connectivity/dimensions, where DMFT becomes exact [4, 66, 67].

[41] G. Rohringer, A. Valli, and A. Toschi, Phys. Rev. B 86, 125114 (2012).

[42] D. Springer, P. Chalupa, S. Ciuchi, G. Sangiovanni, and A. Toschi, Phys. Rev. B 101, 155148 (2020).

[43] $t_{\mathrm{INS}}$ is defined -via the energy-time uncertainty principleas the shortest time-interval accessible to an INS process of maximal energy $E_{\mathrm{INS}}$ of $\mathcal{O}(100 \mathrm{meV})$, consistent to most experiments on the materials considered. $E_{\mathrm{INS}}$ up to $300 \mathrm{meV}$ was achieved in specific cases[34, 36, 68].

[44] T. Kroll, S. Bonhommeau, T. Kachel, H. A. Dürr, J. Werner, G. Behr, A. Koitzsch, R. Hübel, S. Leger, R. Schönfelder, A. K. Ariffin, R. Manzke, F. M. F. de Groot, J. Fink, H. Eschrig, B. Büchner, and M. Knupfer, Phys. Rev. B 78, 220502 (2008).

[45] S. Lafuerza, H. Gretarsson, F. Hardy, T. Wolf, C. Meingast, G. Giovannetti, M. Capone, A. S. Sefat, Y.-J. Kim, P. Glatzel, and L. de' Medici, Phys. Rev. B 96, 045133 (2017).

[46] G. Kresse and J. Hafner, Phys. Rev. B 47, 558 (1993).

[47] G. Kresse and J. Hafner, Phys. Rev. B 49, 14251 (1994).

[48] A. A. Mostofi, J. R. Yates, G. Pizzi, Y.-S. Lee, I. Souza,
D. Vanderbilt, and N. Marzari, Computer Physics Communications 185, 2309 (2014).

[49] C. Watzenböck, Quantum dynamical screening of the local magnetic moment in the different families of Fe-based superconductors, Master's thesis, Vienna University of Technology (2018).

[50] M. T. Czyżyk and G. A. Sawatzky, Phys. Rev. B 49, 14211 (1994)

[51] S. Li, C. de la Cruz, Q. Huang, Y. Chen, J. W. Lynn, J. Hu, Y.-L. Huang, F.-C. Hsu, K.-W. Yeh, M.-K. Wu, and P. Dai, Phys. Rev. B 79, 054503 (2009).

[52] X. Wang, Q. Liu, Y. Lv, Z. Deng, K. Zhao, R. Yu, J. Zhu, and C. Jin, Science China Physics, Mechanics and Astronomy 53, 1199 (2010).

[53] V. Grinenko, M. Abdel-Hafiez, S. Aswartham, A. U. B. Wolter-Giraud, C. Hess, M. Kumar, S. Wurmehl, K. Nenkov, G. Fuchs, B. Holzapfel, S. L. Drechsler, and B. Buechner, arXiv e-prints , arXiv:1203.1585 (2012), arXiv:1203.1585 [cond-mat.supr-con].

[54] A. Kowalski, A. Hausoel, M. Wallerberger, P. Gunacker, and G. Sangiovanni, Phys. Rev. B 99, 155112 (2019).

[55] R. Levy, J. P. F. LeBlanc, and E. Gull, Computer Physics Communications 215, 149 (2017), arXiv:1606.00368 [physics.comp-ph].

[56] J. Otsuki, M. Ohzeki, H. Shinaoka, and K. Yoshimi, Phys. Rev. E 95, 061302 (2017).

[57] J. Pelliciari, Y. Huang, K. Ishii, C. Zhang, P. Dai, G. F. Chen, L. Xing, X. Wang, C. Jin, H. Ding, P. Werner, and T. Schmitt, Scientific Reports 7, 8003 (2017).

[58] Yeh, Kuo-Wei, Huang, Tzu-Wen, Huang, Yi-lin, Chen, Ta-Kun, Hsu, Fong-Chi, Wu, Phillip M., Lee, Yong-Chi, Chu, Yan-Yi, Chen, Chi-Lian, Luo, Jiu-Yong, Yan, DerChung, and Wu, Maw-Kuen, EPL 84, 37002 (2008).

[59] F. Grønvold, H. Haraldsen, and J. Vihodve, Acta Chem. Scand. 8, 1927 (1954).

[60] S. Rózsa and H.-U. Schuster, Zeitschrift fr Naturforschung B 36, 1668 (1981).

[61] M. Rotter, M. Tegel, D. Johrendt, I. Schellenberg, W. Hermes, and R. Pöttgen, Phys. Rev. B 78, 020503 (2008).

[62] J. H. Tapp, Z. Tang, B. Lv, K. Sasmal, B. Lorenz, P. C. W. Chu, and A. M. Guloy, Phys. Rev. B 78, 060505 (2008).

[63] M. J. Pitcher, D. R. Parker, P. Adamson, S. J. C. Herkelrath, A. T. Boothroyd, R. M. Ibberson, M. Brunelli, and S. J. Clarke, Chem. Commun. , 5918 (2008).

[64] Y. Kamihara, T. Watanabe, M. Hirano, and H. Hosono, Journal of the American Chemical Society 130, 3296 (2008), pMID: 18293989, https://doi.org/10.1021/ja800073m.

[65] C. de la Cruz, Q. Huang, J. W. Lynn, J. Li, W. R. II, J. L. Zarestky, H. A. Mook, G. F. Chen, J. L. Luo, N. L. Wang, and P. Dai, Nature 453, 899 EP (2008).

[66] E. Pavarini, E. Koch, D. Vollhardt, and A. I. Lichtenstein, "Dmft at 25: Infinite dimensions: Lecture notes of the autumn school on correlated electrons," (Forschungszentrum Jülich, Zentralbibliothek, Verlag, 2014) Chap. 4, pp. 91-118.

[67] L. Del Re and A. Toschi, (2020), (unpublished).

[68] C. Armstrong, E. Sandqvist, and M. Rheinstadter, Protein Pept. Lett. 18, 344 (2011). 


\title{
Supplemental Material for "Characteristic time-scales of the local moment dynamics in Hund's-metals"
}

\author{
C. Watzenböck ${ }^{a}$, M. Edelmann ${ }^{b}$, D. Springer ${ }^{a}$, G. Sangiovanni ${ }^{b}$, and A. Toschi ${ }^{a 1,2}$ \\ ${ }^{1 a}$ Institute of Solid State Physics, TU Wien, 1040 Vienna, Austria \\ ${ }^{2 b}$ Institut für Theoretische Physik und Astrophysik, Universität Würzburg, \\ Am Hubland Campus Süd, 97074 Würzburg, Germany
}

\section{COMPUTATIONAL DETAILS}

Density Functional Theory - For the density functional theory (DFT) calculations we employed the VASP code[1, 2], version 5.3.3. As structural inputs, the experimentally found crystal structures as well as the measured lattice parameters (given in table I) have been used.

For all of the atoms in the given structures, we used PBE-GGA functionals. The precise functionals used for each atom are given in table II. Calculations were performed on a $\Gamma$-centered MP k-mesh with $12 \times 12 \times 12$ points and $10 \times 10 \times 12$ points for $\mathrm{I} 4 / \mathrm{mmm}$ and $\mathrm{P} 4 / \mathrm{nmm}$ structures, respectively; The partial occupancies were calculated using the Blöchl tetrahedron method. The respective cut-off energies were, among other parameters, defined by setting the precision to HIGH, and the DOS was evaluated on 2001 points.

\begin{tabular}{|c|c|c|c|c|c|}
\hline Material & Crystal structure & Space group & $a[\AA]$ & $c[\AA]$ & $\mathrm{z}$ \\
\hline $\mathrm{LaFeAsO}$ & ZrCuSiAs-type & $\mathrm{P} 4 / \mathrm{nmm}$ & $4.0355[3]$ & $8.7393[3]$ & $0.1418_{\mathrm{La}}, 0.6507_{\mathrm{As}}[4]$ \\
\hline LiFeAs & PbFCl-type tetragonal & $\mathrm{P} 4 / \mathrm{nmm}$ & $3.774[5]$ & $6.354[5]$ & $0.8459_{\mathrm{Li}}, 0.2635_{\mathrm{As}}[6]$ \\
\hline $\mathrm{BaFe}_{2} \mathrm{As}_{2}$ & $\mathrm{ThCr}_{2} \mathrm{Si}_{2}$-type & $\mathrm{I} 4 / \mathrm{mmm}$ & $3.9625[7]$ & $13.0168[7]$ & $0.3545_{\mathrm{As}}[7]$ \\
\hline $\mathrm{KFe}_{2} \mathrm{As}_{2}$ & $\mathrm{ThCr}_{2} \mathrm{Si}_{2}$-type & $\mathrm{I} 4 / \mathrm{mmm}$ & $3.842[8]$ & $13.861[8]$ & $0.3525_{\mathrm{As}}[8]$ \\
\hline $\mathrm{FeTe}$ & PbO-type & $\mathrm{P} 4 / \mathrm{nmm}$ & $3.8279[9]$ & $6.2561[9]$ & $0.285 \mathrm{Te}[10]$ \\
\hline
\end{tabular}

TABLE I: Crystal structures for all materials under consideration. For I4/mmm materials, $c$ is given as the lattice parameter of the tetragonal cell, and $z$ in relation to this $c$.

\begin{tabular}{lll}
\hline Element & Creation date & VHRFIN \\
\hline La & Sep 6th 2000 & core Kr4d \\
Fe & Sep 6th 2000 & d7s1 \\
Ba & Sep 6th 2000 & 5s5p6s \\
Te & Apr 8th 2002 & s2p4 \\
O & Apr 8th 2002 & s2p4 \\
K & Jan 17th 2003 & p6s1 \\
Li & Jan 17th 2003 & s1p0 \\
As & Sep 22nd 2009 & s2p3 \\
\hline
\end{tabular}

TABLE II: List of PAW PBE functionals used by VASP in the DFT calculations of this study. The functionals are uniquely identified by their creation date and the valence electron configuration given in the functionals by VHRFIN.

Wannier Projection - The VASP results were projected onto local orbitals via the wannier90 code[11]. At the time of the calculations, wannier90 integration into VAPS was only possible with wannier90 v1.2. Specifically, all of the electronic Bloch functions in the DFT calculation were projected onto the $d$ states of Fe, no bands were marked as excluded in the wannier90.win file. In wannier90, the k-points were identical to those from the respective VASP MP-grids. The electronic bands of predominant Fe character are intertwined with bands of other character, such as the $p$ states of the ligands. The degree of entanglement varied across materials, necessitating different disentanglement window parameters for wannier90, and in the case of $\mathrm{BaFe}_{2} \mathrm{As}_{2}$ and $\mathrm{KFe}_{2} \mathrm{As}_{2}$ also frozen windows. The window 
positions were tweaked manually with respect to disentanglement convergence as well as agreement between original VASP bands and the bands of the Wannier Hamiltonian, the final values are given in table III. The convergence criterion for the disentanglement as well as the wannierization was a difference in spread between successive iterations lower than $10^{-11}$. Best results were achieved by enabling guiding centres. The Wannier Hamiltonian served as the single-particle Hamiltonian for the Dynamical Mean Field Theory (DMFT) calculations.

\begin{tabular}{lccccc}
\hline \multirow{2}{*}{ Material } & \multicolumn{2}{c}{ Disentangle window $[\mathrm{eV}]$} & \multicolumn{2}{c}{ Frozen window $[\mathrm{eV}]$} \\
\cline { 2 - 3 } & $\min$ & $\max$ & & $\min$ & $\max$ \\
\hline $\mathrm{LaFeAsO}$ & -2.0473 & 2.4527 & & \\
$\mathrm{LiFeAs}$ & -2.4441 & 2.7559 & & \\
$\mathrm{BaFe}_{2} \mathrm{As}_{2}$ & -1.0723 & 2.7277 & -1.0723 & -0.3723 \\
$\mathrm{KFe}_{2} \mathrm{As}_{2}$ & -4.7175 & 3.2825 & -1.3175 & 3.0825 \\
$\mathrm{FeTe}$ & -2.0831 & 2.4169 & & \\
\hline
\end{tabular}

TABLE III: Energy windows for disentangling of bands in wannier90. $\mathrm{KFe}_{2} \mathrm{As}_{2}$ and $\mathrm{BaFe}_{2} \mathrm{As}_{2}$ additionally required windows defining frozen states. Energies are given relative to the Fermi energy $\mathrm{E}_{\mathrm{F}}=0 \mathrm{eV}$.

Dynamical Mean Field Theory - To include the effects of strong local interactions on top of the DFT, we performed DMFT simulations of an low-energy model for the entire $3 d$-orbital manifold of Fe. The most general form of an on-site electrostatic repulsion in this manifold reads

$$
H_{\mathrm{int}}=\sum_{\mathbf{r} \sigma \sigma^{\prime}} \sum_{l m n o} U_{l m n o} c_{\mathbf{r} l \sigma}^{\dagger} c_{\mathbf{r} m \sigma^{\prime}}^{\dagger} c_{\mathbf{r} o \sigma^{\prime}} c_{\mathbf{r} n \sigma},
$$

where the full-fledged, four-indexed $U$-tensor describes the projected value of the screened Coulomb interaction on the corresponding orbital configurations. As an ab-initio estimate for the orbital-dependent interaction parameters, we take the results by Miyake et al.[12], where constrained random phase approximation (cRPA) results for the twoorbital interaction matrix $U_{l m}$ and $J_{l m}$ were reported for different compounds[26]. Here, the $J_{l m}$ values encode the (orbital-dependent) Hund's coupling, while the $U_{i j}$ diagonal/off-diagonal matrix elements describe the inter-/intraorbital electrostatic repulsion. The relation, which we exploited to extract the interaction parameters appearing in Eq. 1, is:

$$
U_{i j k l}= \begin{cases}U_{i j}, & \text { if } i j k l=i j i j, \\ J_{i j}, & \text { if } i j k l=i i j j \text { and } i \neq j, \\ J_{i j}, & \text { if } i j k l=i j j i \text { and } i \neq j, \\ 0, & \text { otherwise. }\end{cases}
$$

This leads to the low-energy Hamilonian used for our DMFT calculations

$$
H=\sum_{\mathbf{k} \sigma l m} H_{l m}(\mathbf{k}) c_{\mathbf{k} l \sigma}^{\dagger} c_{\mathbf{k} m \sigma}+H_{\mathrm{int}}
$$

with

$$
\begin{aligned}
H_{\mathrm{int}}= & \sum_{\mathbf{r} l} U_{l l} n_{\mathbf{r} l \uparrow} n_{\mathbf{r} l \downarrow}+\sum_{\mathbf{r} \sigma \sigma^{\prime}} \sum_{l<m}\left(U_{l m}-J_{l m} \delta_{\sigma \sigma^{\prime}}\right) n_{\mathbf{r} l \sigma} n_{\mathbf{r} m \sigma^{\prime}} \\
& -\sum_{\mathbf{r}} \sum_{l \neq m} J_{l m} c_{\mathbf{r} l \uparrow}^{\dagger} c_{\mathbf{r} l \downarrow}^{\dagger} c_{\mathbf{r} m \uparrow} c_{\mathbf{r} m \downarrow}-\sum_{\mathbf{r}} \sum_{l \neq m} J_{l m} c_{\mathbf{r} l \uparrow}^{\dagger} c_{\mathbf{r} m \downarrow}^{\dagger} c_{\mathbf{r} m \uparrow} c_{\mathbf{r} l \downarrow} .
\end{aligned}
$$

Physically, this corresponds to an orbital-dependent Kanamori interaction, where one can easily recognize an orbitaldependent pair-hopping (the first term in the second line) and spin-flip contribution (second term). In fact, eq. (4) can be regarded as an orbital-dependent generalization of the Kanamori interaction, since for the special cases of no-orbital dependence e.g. averaged interaction parameters (where $U_{l l}=U, U_{l \neq m}=V$ and $J_{l m}=J$ ) we recover the usual expression of the Kanamori Hamiltonian:

$$
\begin{aligned}
H_{\mathrm{int}}^{K}= & U \sum_{\mathbf{r} l} n_{\mathbf{r} l \uparrow} n_{\mathbf{r} l \downarrow}+\sum_{\mathbf{r} \sigma \sigma^{\prime}}\left(V-J \delta_{\sigma \sigma^{\prime}}\right) \sum_{l<m} n_{\mathbf{r} l \sigma} n_{\mathbf{r} m \sigma^{\prime}} \\
& -J \sum_{\mathbf{r}} \sum_{l \neq m} c_{\mathbf{r} l \uparrow}^{\dagger} c_{\mathbf{r} l \downarrow}^{\dagger} c_{\mathbf{r} m \uparrow} c_{\mathbf{r} m \downarrow}-J \sum_{\mathbf{r}} \sum_{l \neq m} c_{\mathbf{r} l \uparrow}^{\dagger} c_{\mathbf{r} m \downarrow}^{\dagger} c_{\mathbf{r} m \uparrow} c_{\mathbf{r} l \downarrow} .
\end{aligned}
$$


To illustrate concisely the variation of the screened interaction values in the different materials, the corresponding orbitally average $U$ - and $J$-values are shown in table IV. The DMFT calculations shown in the main text were, however, performed using the orbital-resolved Hamiltonian (4). Finally, let us mention that in order to check the

\begin{tabular}{lcc}
\hline & $\bar{U}$ & $\bar{J}$ \\
\hline $\mathrm{LaFeAsO}$ & 2.53 & 0.39 \\
$\mathrm{BaFe}_{2} \mathrm{As}_{2}$ & 2.81 & 0.43 \\
$\mathrm{KFe}_{2} \mathrm{As}_{2}$ & 2.81 & 0.43 \\
$\mathrm{LiFeAs}$ & 3.15 & 0.43 \\
$\mathrm{FeTe}$ & 3.41 & 0.48 \\
\hline
\end{tabular}

TABLE IV: Average effective on-site Coulomb $(\mathrm{U})$ exchange $(\mathrm{J})$ interactions between two electrons on the same iron site in the $d$ - model (in eV).

robustness of our conclusions, we have also performed DMFT calculations using the orbitally-averaged values for the $U$ and $J$ interaction (i.e., corresponding to a "conventional" Kanamori interaction, not shown), finding only marginal changes to the results shown in Fig. 2 and 3 of the main text. Larger quantitative modifications can be found in the results of the most correlated materials, as expected, only if one neglects the spin-flip terms in Eq. (5) (e.g., when performing density-density calculations, not shown here[13]). The reason is, that in this approximation one tends to overestimate the high-spin configurations in the strong-coupling regime.

The number of electrons in the target $(d-)$ manifold was estimated directly from chemical considerations (constituent electronegativity). Throughout our calculation, we assumed that LaFeAsO, $\mathrm{BaFe}_{2} \mathrm{As}_{2} \mathrm{LiFeAs}$ and FeTe have a filling of $\sum_{l, \sigma}\left\langle n_{\sigma}^{l}\right\rangle=6.0$ electrons per iron atom. For $\mathrm{KFe}_{2} \mathrm{As}_{2}$ we used, instead, $\sum_{l, \sigma}\left\langle n_{\sigma}^{l}\right\rangle=5.5$ per iron atom.

To avoid double-counting of the Coulomb interaction between Fe-3d electrons already included in DFT, we used an orbital-dependent double-counting correction of the Fully-Localized-Limit (FLL) type [14] (adopted also for DMFT calculations of elemental Fe[15]). The values used were determined by eq. (6) and are shown in table V.

$$
\mu_{D C}^{F L L}(i)=\mu_{D C}^{F L L}(i)+\frac{1}{4}\left(n^{0}-\frac{1}{2}\right)\left(\sum_{j}\left(U_{i j}-J_{i j}\right)\right)
$$

In eq. (6) $n^{0}=\frac{1}{2(2 l+1)} \sum_{i, \sigma} n_{i, \sigma}$ is the DFT filling and the two-indices U-matrix is related to the four-indices local (screened) Coulomb-tensor by $U_{i j}=U_{i j i j}$ and $J_{i j}=U_{i j j i}($ with $i \neq j$ ).

\begin{tabular}{lccccc}
\hline \multicolumn{2}{c}{$\mathrm{LaFeAsO} \mathrm{BaFe}_{2} \mathrm{As}_{2}$} & $\mathrm{LiFeAs}$ & $\mathrm{KFe}_{2} \mathrm{As}_{2}$ & $\mathrm{FeTe}$ \\
\hline $3 z^{2}-r^{2}$ & 9.5295 & 10.8510 & 12.5740 & 9.8805 & 13.3397 \\
$x z$ & 8.9790 & 9.9117 & 11.6900 & 9.0234 & 11.9597 \\
$y z$ & 8.9790 & 9.9117 & 11.6900 & 9.0234 & 11.9597 \\
$x^{2}-y^{2}$ & 8.2157 & 9.4147 & 11.5623 & 8.5699 & 13.2400 \\
$x y$ & 9.6732 & 10.6073 & 12.2523 & 9.6586 & 13.5735 \\
\hline
\end{tabular}

TABLE V: Orbital dependent double counting correction (DCC) in the fully-localized limit.

The DMFT simulation was performed with a continuous-time quantum Monte Carlo (QMC) algorithm implemented in the code package w2dynamics[16]. All calculations were done at $\beta=50\left[\mathrm{eV}^{-1}\right]$ corresponding to approximately $232.1 \mathrm{~K}$. At this temperature all the materials are experimentally found to be in the paramagnetic phase[17-19]. To achieve convergence in the DMFT cycle with orbital dependent Kanamori interaction for the different materials we first converged the DMFT cycle without the pair-hopping term in Eq. (4)). This was achieved by performing $30 \div 70$ DMFT steps with low statistics (Nmeas $=10^{4}$, where Nmeas is then number of QMC measurements, see [16] for details). Up to 100 additional DMFT-steps were performed with higher statistics.

For each calculations, the final convergence of the DMFT self-consistency was tested for the one-particle quantities encoded in the self-energy $\Sigma^{l}(\omega)$, with respect to the previous five iterations. For the number of steps between measurements (where the minimum value gives a measure of auto-correlation time) we found a value of $\mathrm{Ncorr}=1500 \div$ 2000 to be sufficient, in line with the estimate of the average "renewal time" of the fermionic trace given in Ref.16.

On top of the converged one-particle quantities we then calculated the spin-spin susceptibility in imaginary time through a single DMFT step with fixed chemical potential and one-particle properties, using $\mathrm{Nmeas}>5 \cdot 10^{4}$. As a 
QMC-sampling algorithm we applied the recently developed States-Sampling [20]. The result is show in the upperright part of Figure 2 of the main text.

To obtain the DMFT-bubble susceptibility we used $\chi^{\text {Bubble-DMFT }}(\tau)=\sum_{l} G_{\text {loc }}^{l}(\tau) G_{\text {loc }}^{l}(\beta-\tau)$, and for the barebubble we set the interaction as well as the double counting correction in the DMFT calculation to zero $(U=J=$ $\left.V=\mu_{D C}=0\right)$, corresponding to $G_{\mathrm{loc}}=G_{0}$.

Analytical continuation - Analytical continuation from imaginary time (where the QMC data was obtained) to real frequencies was performed with the Maximum Entropy Method (MaxEnt) with the code package Maxent [21]. This way we obtained the imaginary part of the retarded susceptibility $\chi^{R}(t) \equiv \frac{\mathrm{i}}{\hbar} \theta(t)\left\langle\left[\hat{S}_{z}(t), \hat{S}_{z}(0)\right]\right\rangle$. The effect of different default-models (Flat, Gaussian, Lorentzian) was tested and found to be small. We chose a broad featureless Lorentziandefault model with a width of $\gamma_{\text {Model }}=0.5$. Model-details are found elsewhere [21]. The optimal $\alpha$-parameter (weight of the entropy term in MaxEnt) was determined by the maximum of the curvature of $\chi^{2}(\alpha)$. (See fig. 1.) This way we could reliably determine the region where neither the data was over-fitted nor the default model was take into account too strongly. One advantage of this method is invariance of the final spectrum under re-scaling the error by a global factor[13]. A similar approach was already applied in [22].
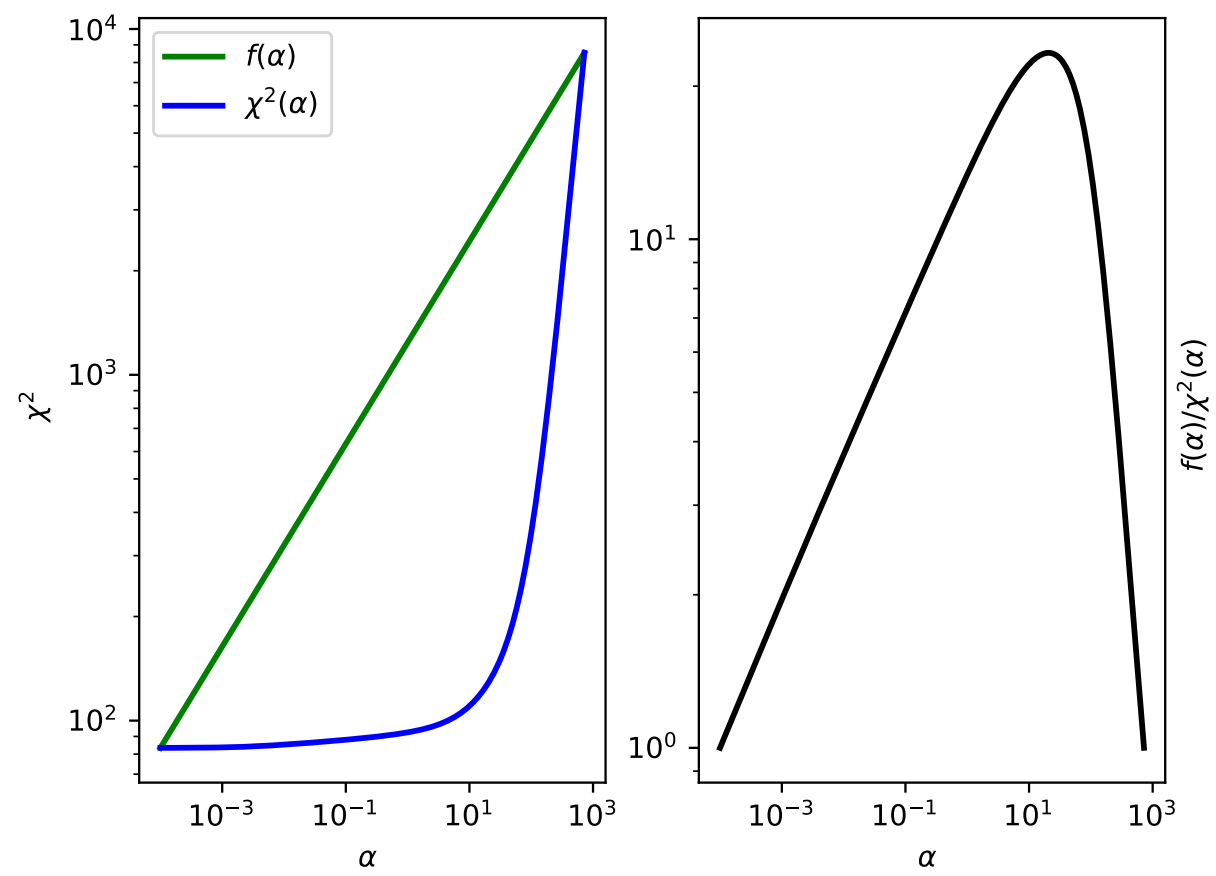

FIG. 1: Log-log-plot of the quadratic difference between the data and the fit $\chi^{2}$ over the entropy parameter $\alpha$ for LaFeAsO. We find overfitting (underfitting) of the data to start at $\alpha<10^{0}\left(\alpha>10^{2}\right)$. The spectrum corresponding to the maximum of $f(\alpha) / \chi^{2}(\alpha)\left(\right.$ at $\left.\alpha=2 \cdot 10^{1}\right)$ could be regarded as a good analytical continuation. 
One particle time scales- For estimating one particle timescales we assumed that (in the presence of a well defined quasi-particle excitation) the one-particle Green's function $G^{i}(t)$ for each orbital $i$ decays in the following way: $\left|G^{i}(t)\right|^{2}=Z_{i}^{2} \mathrm{e}^{-\frac{t 2 Z_{i} \operatorname{Im} \Sigma_{i}(\omega \rightarrow 0)}{\hbar}} \propto \mathrm{e}^{-\frac{t}{t_{1 \mathrm{P}}^{i}}}$, with $t_{1 \mathrm{P}}^{i} \equiv \frac{\hbar}{2 Z_{i} \operatorname{Im} \Sigma_{i}(\omega \rightarrow 0)}$, where $\Sigma_{i}$ is the self-energy of the orbital $i$. The value of the self-energy at zero frequency as well as the orbital dependent quasi-particle mass re-normalization $Z_{i}=\left(1+\mathrm{d} /\left.\mathrm{d} \omega \operatorname{Re} \Sigma_{i}(\omega)\right|_{\omega \rightarrow 0}\right)^{-1}$ was extracted from the DMFT self-energy by linear interpolation of $\operatorname{Im} \Sigma\left(\mathrm{i} \omega_{n} \rightarrow 0\right)$ (using the Cauchy-Riemann equations for $Z_{i}$ ). The one particle time scale $t_{1 \mathrm{P}}$ given in the main text was then estimated as the orbital average of $t_{1 \mathrm{P}}^{i}: t_{1 \mathrm{P}}=\frac{1}{5} \sum_{i} t_{1 \mathrm{P}}^{i}$.

Spin-excitation time scales - While the time scales of spin-excitations in iron-based superconductors are determined by an intricate interplay of kinetic energy (hopping) and electron-electron-interaction the main time scales can be effectively described by a much simpler model. The extraction of time scales was done by applying a uniform $\chi^{2}$-fit to $\operatorname{Im} \chi^{R}(\omega)$ with cutoff-values chosen for the grid such that the main-peaks structure is well within the frequency window $(1 \mathrm{eV})$. The cutoff excludes high-frequency data, which is usually not as well captured by MaxEnt as the low-frequency data. A variation of the cutoff by $20 \%$ leads to a change in the time scales by less than $15 \%$.

The fitting function is defined as follows: We consider the absorption spectrum of a damped harmonic oscillator, which can be obtained by the Fourier-transform of the Green's function of the differential equation $\ddot{\chi}(t)+2 \gamma \dot{\chi}(t)-$ $\omega_{0}^{2} \chi(t)=-\delta(t)$, i.e. $\chi(\omega)=\frac{1}{\omega^{2}-2 \mathrm{i} \gamma \omega+\omega_{0}^{2}}$. We note that the latter has poles only on the lower half-plane, and thus it is a retarded function $(\chi(t<0)=0$ ). Its imaginary part (up to a proportionality-constant reflecting the material-dependent value of the unscreened local moment) defines our fitting model which, thus, reads

$$
\operatorname{Im} \chi^{R}(\omega)=2 \gamma \omega \frac{1}{\left(\omega^{2}-\omega_{0}^{2}\right)^{2}+4 \omega^{2} \gamma^{2}},
$$

or correspondingly in real times

$$
\chi(t)= \begin{cases}\frac{\mathrm{e}^{-\gamma t}}{\sqrt{\omega_{0}^{2}-\gamma^{2}}} \sin \left(\sqrt{\omega_{0}^{2}-\gamma^{2}} t\right) \theta(t) & \text { if } \omega_{0}^{2}>\gamma^{2} \\ \frac{\mathrm{e}^{-\gamma t}}{\sqrt{\gamma^{2}-\omega_{0}^{2}}} \sinh \left(\sqrt{\gamma^{2}-\omega_{0}^{2}} t\right) \theta(t) & \text { if } \omega_{0}^{2}<\gamma^{2} .\end{cases}
$$

The asymptotic behavior, which determines the main-lifetime is given by

$$
\lim _{t \rightarrow \infty} \chi(t) \propto\left\{\begin{array}{lll}
\mathrm{e}^{-\gamma t} & \equiv \mathrm{e}^{-t / t_{\gamma}^{\text {under }}} & \text { if } \omega_{0}^{2}>\gamma^{2} \\
\mathrm{e}^{-\left(\gamma-\sqrt{\gamma^{2}-\omega_{0}^{2}}\right) t} & \equiv \mathrm{e}^{-t / t_{\gamma}^{\text {over }}} & \text { if } \omega_{0}^{2}<\gamma^{2} .
\end{array}\right.
$$

\begin{tabular}{|c|c|c|c|c|}
\hline & $\omega_{0}[\mathrm{eV}]$ & $\gamma[\mathrm{eV}]$ & $t_{\gamma}[\mathrm{fs}]$ & $t_{\bar{\omega}}[\mathrm{fs}]$ \\
\hline LaFeAsO & 0.39 & 0.35 & 1.9 & 3.8 \\
\hline $\mathrm{BaFe}_{2} \mathrm{As}_{2}$ & 0.28 & 0.28 & 2.4 & 15.2 \\
\hline LiFeAs & 0.30 & 0.58 & 7.9 & - \\
\hline $\mathrm{KFe}_{2} \mathrm{As}_{2}$ & 0.51 & 2.08 & 10.3 & - \\
\hline $\mathrm{FeTe}$ & 0.029 & 0.022 & 29.3 & 34.8 \\
\hline
\end{tabular}

The corresponding parameters obtained by fitting the DMFT spectra are summarized in table VI.

TABLE VI: Fitting parameters extracted with a harmonic oscillator model (second and third column), effective lifetime $\chi(t \rightarrow$ $\infty) \propto \mathrm{e}^{-t / t_{\gamma}}$ (third column) and effective oscillation frequency $t_{\bar{\omega}}=\frac{\hbar}{\sqrt{\omega_{0}^{2}-\gamma^{2}}}$ (fourth column)

One can also define a harmonic-oscillator anti-commutator through the fluctuation-dissipation theorem as $F(\omega)=$ $\frac{1}{\pi} \operatorname{coth}(\omega \beta / 2) \operatorname{Im} \chi^{R}(\omega)$. For the latter it is not easy to get an analytical expression for the Fourier-transform $\left(F^{\text {harm. osz. }}(t)=\int_{-\infty}^{\infty} \mathrm{d} \omega \mathrm{e}^{-\mathrm{i} \omega t} \frac{1}{\pi} \operatorname{coth}(\omega \beta / 2)\right)$. Therefore we preformed the transformation only numerically. To assess the quality of the fit we checked also $\chi^{\text {data }}(t)$ against the analytical expressions given in eq. (8). The results of the transformation of the data as well as the transformation of the fits is shown in fig. 2. For $\mathrm{LaFeAsO}, \mathrm{BeFe}_{2} \mathrm{As}_{2}$, LiFeAs and $\mathrm{KFe}_{2} \mathrm{As}_{2}$ a single peak model was used, while for FeTe the double-peak-structure in the data necessitated a two-peak model. Due to the second peak in FeTe no sign-change is observed in the corresponding $\chi(t)$, although the main (first) peak would predict an oscillatory (under-damped) behavior. 

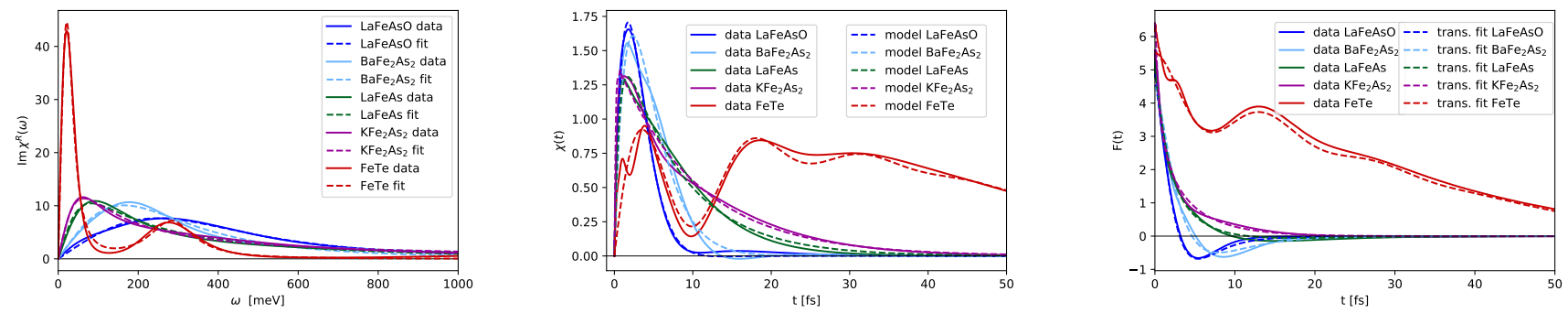

FIG. 2: Dissipative part of the spin-spin susceptibility obtained by MaxEnt (left figure solid lines). The harmonic-oscillator fits are shown as dashed lines. Center figure: Spin-spin susceptibility in time. Direct transform of MaxEnt data shown as solid lines and the analytic expression for the fitted model as dashed lines. Right figure: Spin-spin anti-commutator correlation function for data (solid lines) and fitted model (dashed lines).

A comparison between the right and the center-part of fig. 2 shows the same behavior qualitatively, although quantitative differences are observed. One reason for the difference is the additional energy scale (temperature).

Comments on previous works - The estimated values of the local fluctuating moment $\left\langle m_{\mathrm{loc}}^{2}\right\rangle$ in the specific case of $\mathrm{KFe}_{2} \mathrm{As}_{2}$ obtained by our DFT+DMFT study deviates from the results of [23], where $\left\langle m^{2}\right\rangle=0.1 \pm 0.02\left[\mu_{B}^{2} / \mathrm{Fe}\right]$. According to our work $\mathrm{KFe}_{2} \mathrm{As}_{2}$ should not have a significantly different local magnetic moment compared with the other iron-pnictides/-chalcogenides, which is consistent with the more recent theoretical/experimental analysis of $[24,25]$.

[1] G. Kresse and J. Hafner, Phys. Rev. B 47, 558 (1993), URL http://link.aps.org/doi/10.1103/PhysRevB.47.558.

[2] G. Kresse and J. Hafner, Phys. Rev. B 49, 14251 (1994), URL http://link.aps.org/doi/10.1103/PhysRevB.49.14251.

[3] Y. Kamihara, T. Watanabe, M. Hirano, and H. Hosono, Journal of the American Chemical Society 130, 3296 (2008), pMID: 18293989, https://doi.org/10.1021/ja800073m, URL https://doi.org/10.1021/ja800073m.

[4] C. de la Cruz, Q. Huang, J. W. Lynn, J. Li, W. R. II, J. L. Zarestky, H. A. Mook, G. F. Chen, J. L. Luo, N. L. Wang, et al., Nature 453, 899 EP (2008), URL https://doi.org/10.1038/nature07057.

[5] M. J. Pitcher, D. R. Parker, P. Adamson, S. J. C. Herkelrath, A. T. Boothroyd, R. M. Ibberson, M. Brunelli, and S. J. Clarke, Chem. Commun. pp. 5918-5920 (2008), URL http://dx.doi.org/10.1039/B813153H.

[6] J. H. Tapp, Z. Tang, B. Lv, K. Sasmal, B. Lorenz, P. C. W. Chu, and A. M. Guloy, Phys. Rev. B 78, 060505 (2008), URL https://link.aps.org/doi/10.1103/PhysRevB.78.060505.

[7] M. Rotter, M. Tegel, D. Johrendt, I. Schellenberg, W. Hermes, and R. Pöttgen, Phys. Rev. B 78, 020503 (2008), URL https://link.aps.org/doi/10.1103/PhysRevB.78.020503.

[8] S. Rózsa and H.-U. Schuster, Zeitschrift fr Naturforschung B 36, 1668 (1981), URL https://www.degruyter.com/view/ journals/znb/36/12/article-p1668.xml.

[9] Yeh, Kuo-Wei, Huang, Tzu-Wen, Huang, Yi-lin, Chen, Ta-Kun, Hsu, Fong-Chi, Wu, Phillip M., Lee, Yong-Chi, Chu, YanYi, Chen, Chi-Lian, Luo, Jiu-Yong, et al., EPL 84, 37002 (2008), URL https://doi.org/10.1209/0295-5075/84/37002.

[10] F. Grønvold, H. Haraldsen, and J. Vihodve, Acta Chem. Scand. 8, 1927 (1954), URL https://link.aps.org/doi/10. 3891/acta. chem. scand.08-1927.

[11] A. A. Mostofi, J. R. Yates, G. Pizzi, Y.-S. Lee, I. Souza, D. Vanderbilt, and N. Marzari, Computer Physics Communications 185, 2309 (2014), ISSN 0010-4655, URL http://www.sciencedirect.com/science/article/pii/S001046551400157X.

[12] T. Miyake, K. Nakamura, R. Arita, and M. Imada, Journal of the Physical Society of Japan 79, 044705 (2010).

[13] C. Watzenböck, Master's thesis, Vienna University of Technology (2018), URL https://resolver.obvsg.at/urn:nbn:at: at-ubtuw: 1-111991.

[14] M. T. Czyżyk and G. A. Sawatzky, Phys. Rev. B 49, 14211 (1994), URL https://link.aps.org/doi/10.1103/PhysRevB. 49.14211.

[15] A. Hausoel, M. Karolak, E. Şaşıŏlu, A. Lichtenstein, K. Held, A. Katanin, A. Toschi, and G. Sangiovanni, Nature Communications 8, 16062 (2017).

[16] M. Wallerberger, A. Hausoel, P. Gunacker, A. Kowalski, N. Parragh, F. Goth, K. Held, and G. Sangiovanni, Computer Physics Communications 235, 388 (2019), ISSN 0010-4655, URL http://www.sciencedirect.com/science/article/ $\mathrm{pii/S0010465518303217.}$

[17] X. Wang, Q. Liu, Y. Lv, Z. Deng, K. Zhao, R. Yu, J. Zhu, and C. Jin, Science China Physics, Mechanics and Astronomy 53, 1199 (2010), ISSN 1869-1927, URL https://doi.org/10.1007/s11433-010-4031-0.

[18] S. Li, C. de la Cruz, Q. Huang, Y. Chen, J. W. Lynn, J. Hu, Y.-L. Huang, F.-C. Hsu, K.-W. Yeh, M.-K. Wu, et al., Phys. Rev. B 79, 054503 (2009), URL https://link.aps.org/doi/10.1103/PhysRevB.79.054503. 
[19] V. Grinenko, M. Abdel-Hafiez, S. Aswartham, A. U. B. Wolter-Giraud, C. Hess, M. Kumar, S. Wurmehl, K. Nenkov, G. Fuchs, B. Holzapfel, et al., arXiv e-prints arXiv:1203.1585 (2012), 1203.1585.

[20] A. Kowalski, A. Hausoel, M. Wallerberger, P. Gunacker, and G. Sangiovanni, Phys. Rev. B 99, 155112 (2019), URL https://link.aps.org/doi/10.1103/PhysRevB.99.155112.

[21] R. Levy, J. P. F. LeBlanc, and E. Gull, Computer Physics Communications 215, 149 (2017), 1606.00368.

[22] J. Otsuki, M. Ohzeki, H. Shinaoka, and K. Yoshimi, Phys. Rev. E 95, 061302 (2017), URL https://link.aps.org/doi/ 10.1103/PhysRevE.95.061302.

[23] M. Wang, C. Zhang, X. Lu, G. Tan, H. Luo, Y. Song, M. Wang, X. Zhang, E. A. Goremychkin, T. G. Perring, et al., Nature Communications 4, 2874 (2013).

[24] S. Lafuerza, H. Gretarsson, F. Hardy, T. Wolf, C. Meingast, G. Giovannetti, M. Capone, A. S. Sefat, Y.-J. Kim, P. Glatzel, et al., Phys. Rev. B 96, 045133 (2017), URL https://link.aps.org/doi/10.1103/PhysRevB.96.045133.

[25] J. Pelliciari, Y. Huang, K. Ishii, C. Zhang, P. Dai, G. F. Chen, L. Xing, X. Wang, C. Jin, H. Ding, et al., Scientific Reports 7, 8003 (2017), ISSN 2045-2322, URL https://doi.org/10.1038/s41598-017-07286-6.

[26] With the only exception of $\mathrm{KFe}_{2} \mathrm{As}_{2}$, where we used the same values as for the other family-compound (BaFe $\mathrm{As}_{2}$ given in [12]) as they are not available in the literature. 